\title{
RESPONSE OF TEMPERATURE OSCILLATIONS IN A TIN MELT TO CENTRIFUGAL EFFECTS
}

\author{
W.J. Ma, ${ }^{1}$ F. Tao, ${ }^{1}$ Y. Zheng, ${ }^{1}$ M.L. Xue, ${ }^{1}$ B.J. Zhou ${ }^{2}$ and L.Y. Lin ${ }^{2}$ \\ ${ }^{1}$ Institute of Mechanics \\ Chinese Academy of Sciences \\ Beijing 100080, China \\ ${ }^{2}$ Institute of Semiconductors \\ Chinese Academy of Sciences \\ Beijing 100083, China
}

\begin{abstract}
An experimental study was conducted on the flow and temperature oscillations of molten tin in a horizontal boat under centrifugation. A longitudinal temperature gradient was applied to an open boat containing molten tin so that convection was generated with a known direction of the basic flow. The experimental results demonstrated that both effects of centrifugal and Coriolis forces exist due to the rotation of the centrifuge. The former enhances the convection, decreasing the temperature gradient in the melt. The latter shows different influences on the flow stability depending on the rotation sense of the centrifuge. Temperature fluctuations in the melt are considerably retarded, provided the centrifuge rotation is in the same sense as the convection roll in the melt.
\end{abstract}

\section{INTRODUCTION}

Melt growth of crystals is widely used in industry. During the crystal growth process, convection exists within the melt. Convection may be beneficial for transport of heat and mass, but unsteady convection causes temperature fluctuations in the melt. These, in turn, give rise to a fluctuating concentration of solute in the crystal, forming so-called impurity striations. In order to avoid such convection-induced microinhomogeneities, various measures have been taken to maintain a steady state of buoyancy convection or suppress unsteady convection, such as changes of the melt dimension and geometry, reduction of the temperature gradient, application of static magnetic fields, and reduction of gravity (space experiments). High gravity produced by a centrifuge has been used to grow crystal in recent years. Suppression of unsteady convection and doping striations with centrifugal 
acceleration was reported for upside-down vertical Bridgman growth and for horizontal zone melting on a centrifuge. ' We have grown GaAs single crystals by a horizontal gradient freeze method on a large centrifuge. Our preliminary results demonstrated that impurity striations in GaAs grown under centrifugal acceleration became weak and indistinct. ${ }^{2}$ In order to make clear the effects of centrifugation on crystal growth, an experiment was conducted to simulate temperature oscillations in the GaAs melt. In this paper we address the preliminary experimental results for temperature fluctuations of liquid tin, whose $\mathrm{Pr}$ number is similar to that of molten GaAs. The liquid tin was held in a horizontal boat mounted on a centrifuge. Both centrifugal and Coriolis forces had significant influences on the melt flow.

\section{EXPERIMENTAL ARRANGEMENT}

The experiment was carried out on the same centrifuge as described earlier. ${ }^{2}$ It has an arm with a length of $7 \mathrm{~m}$ and an available centrifugal acceleration up to 30 times earth's gravity g. A horizontal cylindrical furnace was hung on the end of the arm via a bearing with its axis perpendicular to both the centrifuge arm and earth's gravity (see Fig. 1 of reference 2). A sealed quartz tube containing an open boat was located in a resistance heater, which established a longitudinal temperature gradient. A tin melt $80 \mathrm{~mm}$ long and $8 \mathrm{~mm}$ high was placed in the boat. A temperature gradient was applied so that thermal convection was generated with the flow ascending at the end with higher temperature and descending at the end with lower temperature. Thermocouples with protectively coated beads were dipped into the melt and fixed at the positions shown in Fig. 1. The temperature gradient and temperature fluctuations in the tin melt were measured by thermocouples $\mathrm{A}, \mathrm{B}$ and $\mathrm{C}(\mathrm{NiSi}-\mathrm{NiCr}$, diameter $1 \mathrm{~mm})$. The temperature gradient $\mathrm{G}$ is defined by the temperature difference between two thermocouples $\mathrm{A}$ and $\mathrm{B}$ divided by the distance between them. The temperature in the furnace was monitored by the thermocouple $\mathrm{M}$ and controlled by a control unit during the whole experiment. The temperature profile in the melt was determined by the furnace temperature and influenced by the rotation of the centrifuge. The power and the amplified thermocouple signals were transmitted via slip rings.

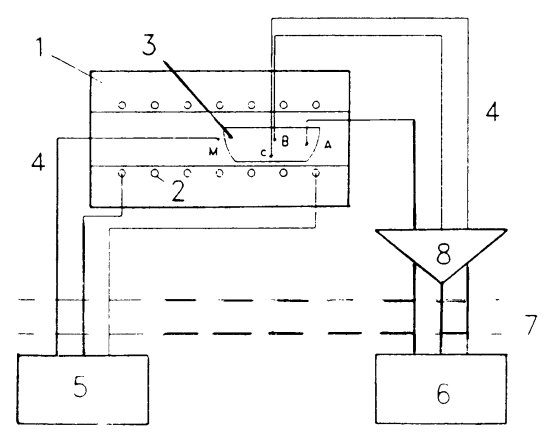

Figure 1. Sketch of the experimental set-up. 1 furnace, 2 resistance heater, 3 boat, 4 thermocouple, 5 thermal control unit, 6 A/D \& chart recorder, 7 slip rings, 8 amplifier. 


\section{RESULTS AND DISCUSSION}

Before running the centrifuge the furnace was heated and the temperature gradient in the furnace increased. The temperature fluctuations in the melt were pronounced, as shown in Fig. 2, which is a plot of the temperature measured at point $\mathrm{C}$ in Fig. 1 for different temperature gradients in the melt. This is consistent with the well-known argument that an increase of the longitudinal temperature gradient will produce temperature fluctuations with increasing amplitude and frequency. ${ }^{3}$

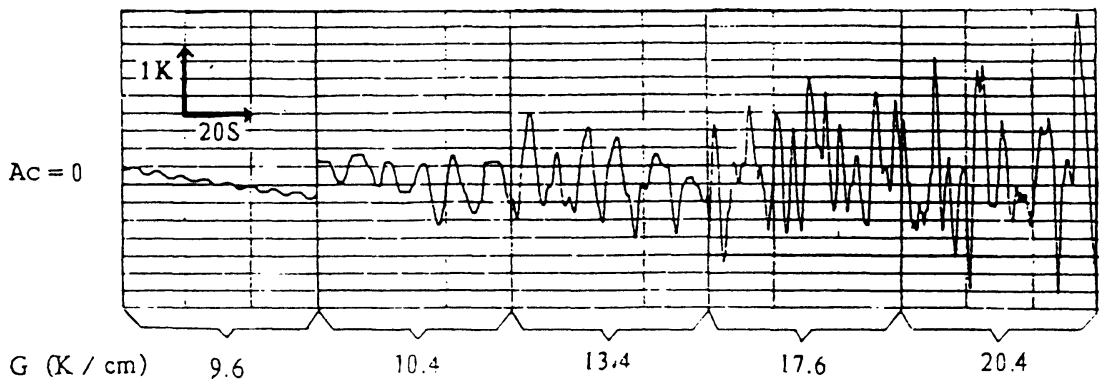

Figure 2. Temperature records for various temperature gradients $\mathrm{G}$ in the melt, without centrifuge rotation. $\mathrm{G}$ is calculated based on the measured temperature difference between thermocouples $\mathrm{A}$ and $\mathrm{B}$.

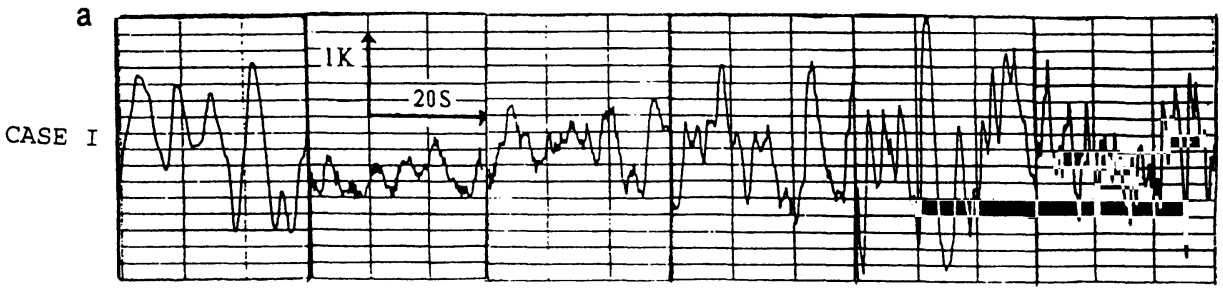

Ac
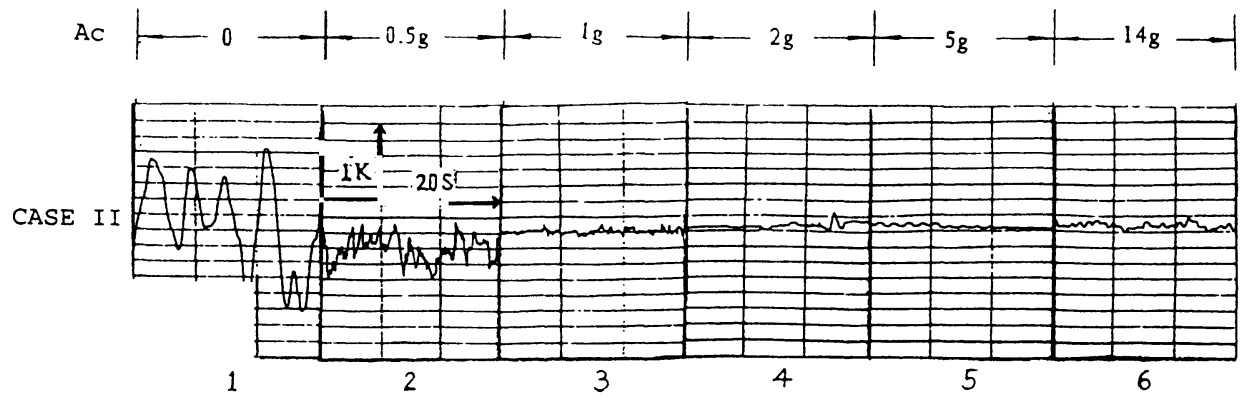

Figure 3. Records of temperature fluctuations (a), and the power spectra (b), for two different rotation senses of the centrifuge. $A_{c}$ is centrifugal acceleration, $g$ is earth's gravity. (continued next page)

When the temperature gradient in the melt reached about $16 \mathrm{~K} / \mathrm{cm}$, the centrifuge was turned on and the rotation rate was raised stepwise, with each step stabilized for about ten minutes. The axial temperature gradient along the furnace was kept constant. Two series of tests were performed with respect to the rotation sense of the centrifuge. Figure 3 is a 
plot of temperature fluctuations measured by thermocouple $\mathrm{C}$ for different centrifugal accelerations and their power spectra for two different rotation senses of the centrifuge. It is seen from Fig. 3(a) that for case I (the direction of the convective roll in the melt is opposite to that of the centrifuge rotation), the amplitude of temperature oscillations increases with increasing centrifugal acceleration $\mathrm{A}_{c}$, and the oscillations of higher frequencies also increase Fig. 3(b). For case II (both directions of the melt convective roll and the centrifuge rotation are in the same sense), the temperature oscillations are dramatically suppressed when $A_{c}>1 g$.
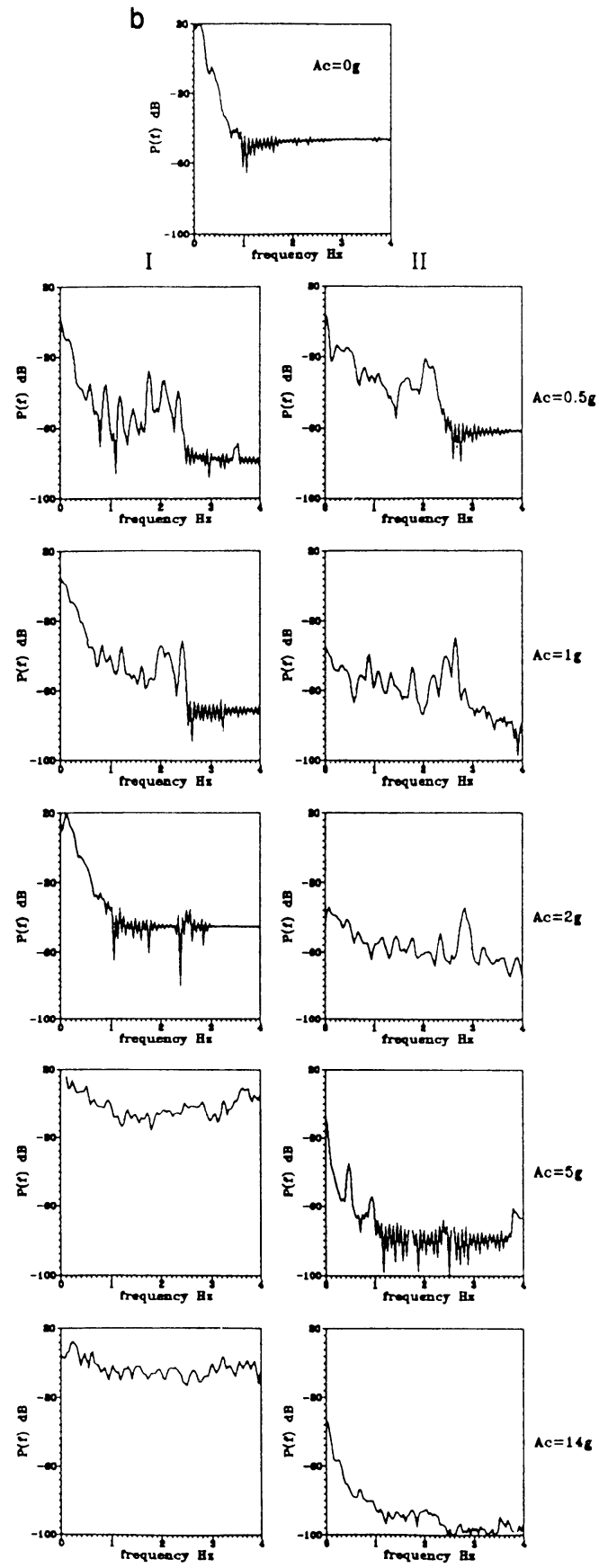

Figure 3. (Continued) 
Figure 4 shows the measured temperature gradient $\mathrm{G}$ in the melt versus the centrifugal acceleration $A_{c}$. In both cases the temperature gradient decreased with increasing centrifugal acceleration. This means that the centrifugal force acts as an increased gravity, enhancing the buoyancy-driven convection and heat transfer, and decreasing the temperature gradient in the melt. It is also seen that for case II the temperature gradients decrease much faster than for case I. The reason will be discussed later.

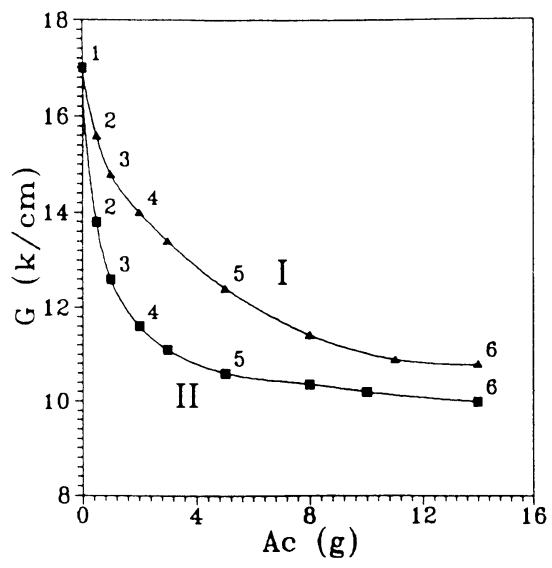

Figure 4. Measured temperature gradient $G$ in the melt versus centrifugal acceleration $A_{c}$ for two cases. The numbered points correspond to those labeled in Fig. 3.

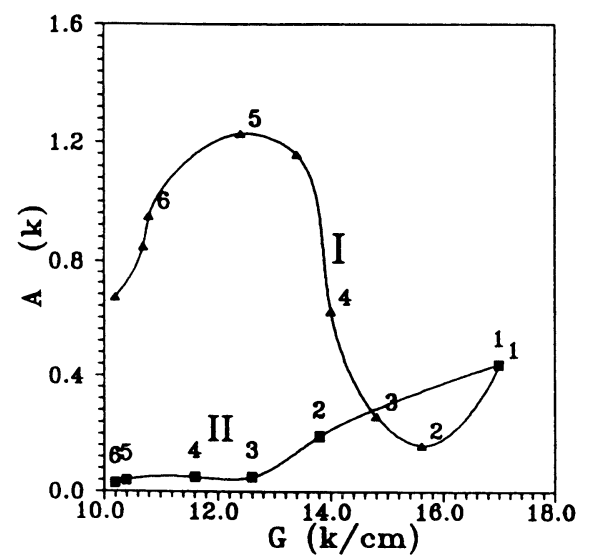

Figure 5. Measured average amplitude $A$ of temperature fluctuations versus temperature gradient $G$ in the melt for two cases. The numbered points correspond to those labeled in Fig. 3.

In Fig. 5 we plot the average amplitude of temperature fluctuations versus the temperature gradient for two different rotation senses. The temperature fluctuations decreased in the initial stage of rotation of the centrifuge for both cases I and II (point 1 to 2 in Fig. 5). The fluctuations continued to decrease with decreasing temperature gradient 
for case II, but increased for case I after point 2. The dependence of the experimental results on the rotation sense of the centrifuge may be explained by the influence of the Coriolis force as stated by Müller et al. ${ }^{1,4}$ For case II the component of the angular velocity of the centrifuge perpendicular to the resultant acceleration vector produces a component of Coriolis force that acts outwards with respect to the convection roll, forcing the fluid to stream along elongated paths. As a result, heat transport is increased and temperature oscillations are damped. For case I, however, the Coriolis force pushes the fluid towards the center of the convection roll causing the flow to become more unstable. By comparing Fig. 5 with Fig. 2, one can find that even for the same temperature gradient G, the amplitude of the oscillations for case II are still smaller than without rotation.

Unlike the vertical Bridgman geometry with top seeding, the horizontal Bridgman configuration has a pre-known direction of the main convection roll subject to the applied temperature gradient. Therefore no bifurcation phenomenon ${ }^{4}$ occurs. This allows one to suppress unwanted fluctuations of temperature during crystal growth by setting the orientation of the furnace or selecting the rotation sense of the centrifuge as required for case II. It should be mentioned that in the horizontal zone melting configuration there are two opposite convective rolls. Both cases I and II may exist simultaneously. Thus a proper orientation of the melt zone (i.e. the zone part adjacent to the growing crystal front is in the situation of case II) seems necessary.

\section{CONCLUDING REMARKS}

The experimental results demonstrate that there are two effects that occur due to the rotation of the centrifuge. One is the effect of the centrifugal force, which enhances the convection and decreases the temperature gradient. Another is the effect of Coriolis force, which leads to different influences on the temperature oscillations depending on the rotation senses of the centrifuge and the convection roll in the melt. This fact has implications for improving crystal growth. A further experiment with fixed thermal conditions at the boat ends and a larger acceleration range is planned.

\section{Acknowledgments}

This work was supported by the National Natural Sciences Foundation and the special financial aid provided by the Chinese Academy of Science. The authors acknowledge BISEE for offering the use of the centrifuge facility.

\section{References}

1. G.Müller, G.Neumann and W.Weber, J. Crystal Growth 119:8 (1992).

2. X.R.Zhong, B.J.Zhou, Q.M.Yan, F.N.Cao, C.J.Li, L.Y.Lin and W.J.Ma, Y.Zheng, F.Tao,M.L.Xue, J. Crystal Growth 119:74 (1992).

3. G.S.Cole, W.C.Winegard, J. Inst. Metals 93:153 (1964).

4. W.Weber, G.Neumann and G.Müller, J. Crystal Growth 100:145 (1990). 